\title{
QUIMIOTERAPIA COMBINADA NEOADYUVANTE SEG UIDA DE RADIOTERAPIA EXTERNA EN EL TRATAMIENTO DE DOS CASOS DE LINFOMA PRIMARIO DEL CUEШO UTERINO
}

\author{
Marco Bravo S. ${ }^{1}$, Fernando Heredia M. ${ }^{1}$, Mateo Pierotic C. ${ }^{1}$ \\ ${ }^{1}$ Unidad de Oncología Ginecológica. Instituto Clínico Oncológico, Fundación Arturo López Pérez.
}

\section{RESUMEN}

Los linfomas primarios del cuello uterino son poco frecuentes. Dado que no hay esquemas de manejo definido, presentamos 2 nuevos casos tratados y controlados desde 1999 a la fecha en la Fundación Arturo López Pérez. El tratamiento consistió en quimioterapia combinada neoadyuvante seguida de radioterapia externa. Se verificó respuesta clínica y radiológica completa en ambos casos. Conclusión: Se puede lograr respuesta completa de estas neoplasias linfoides con esta modalidad de tratamiento.

PALABRAS CLAVE: Linfoma cervical primario

\section{SUMMARY}

Background: Primary extranodal lymphomas of the genital tract are rare. Cases: As there is no current consensus in its management, we present two further cases and their treatment with neoadyuvant chemotherapy, followed by radiation therapy. A radical hysterectomy with bilateral pelvic lymphadenectomy was performed after primary treatment in one case. Clinical response was complete in both cases and pathological response was documented in one. Conclusions: Complete response of these lymphoid neoplasms can be achieved by neoadyuvant chemotherapy followed by external irradiation.

\section{KEY WORDS: Primary cervical lymphoma}

\section{INTRODUCCIÓN}

El aparato genital femenino es un sitio primario infrecuente para un linfoma. Se acepta que 1 de cada 175 linfomas extranodales en mujeres se origina en la vagina, cuerpo o cuello uterino (1). Considerando que no hay consenso en lo que respecta a su manejo, se han intentado diversos protocolos de tratamiento, con resultados igualmente diversos (1-10).

El objetivo de esta comunicación es presentar 2 casos de linfoma primario del cuello uterino tra- tados con quimioterapia neoadyuvante seguida de radioterapia pélvica, lográndose respuesta clínica y radiológica completa en ambos casos.

Caso clínico 1. Paciente de 32 años, nuligesta, que consultó en agosto de 1999 con historia de flujo vaginal sanguinolento y sinusorragia de 2 meses y medio de evolución. Su último examen de citología cervical había sido informado como normal. Al examen genital destacó la presencia de un tumor endofítico cervical de $6 \mathrm{~cm}$ de diámetro mayor. El tumor invadía el tercio superior de la vagina $y$ ambos parametrios en su aspecto 
proximal. Se diagnosticó un tumor en estadío llb de la FIGO. Se tomó biopsia, la que fue preliminarmente informada como carcinoma indiferenciado de células pequeñas o linfoma. Se solicitó estudio de inmunohistoquímica para complementar el diagnóstico histológico. La tomografía axial computada (TAC) de tórax, abdomen y pelvis no mostró evidencias de diseminación tumoral, encontrando solamente el tumor descrito. Dos días más tarde, la paciente consultó por sangramiento masivo el que pudo ser controlado mediante una ligadura extraperitoneal de las arterias hipogástricas mediante 2 incisiones separadas de $4 \mathrm{~cm}$ cada una. La paciente evolucionó de manera satisfactoria. El estudio inmunohistoquímico mostró positividad para CD20 y antígeno leucocitario común, mientras que fue negativo para CD30, CD45Ro y pancitokeratina. De esta manera, el diagnóstico histológico definitivo fue de linfoma no-Hodgkin cervical primario difuso mixto de células grandes y pequeñas, estirpe $B$, de acuerdo a la clasificación de la WHO. La biopsia de médula ósea fue negativa para infiltración medular. Se etapificó como Etapa I-EA de Ann-Arbor. Se trató con 3 ciclos de $\mathrm{CHOP}$, remitiendo completamente, tanto clínica como a la TAC. Tras la quimioterapia se sometió a radioterapia externa con Cobalto 60 (técnica de "four-field box"), completando 4500cGy. Se mantiene sin evidencia de enfermedad 70 meses después del diagnóstico.

Caso clínico 2. Paciente de 31 años, multípara de 1, que consultó en julio de 2003 por hemorragia genital intermitente de 2 meses de evolución. Su último examen de citología cervical había sido informado como normal. El examen ginecológico

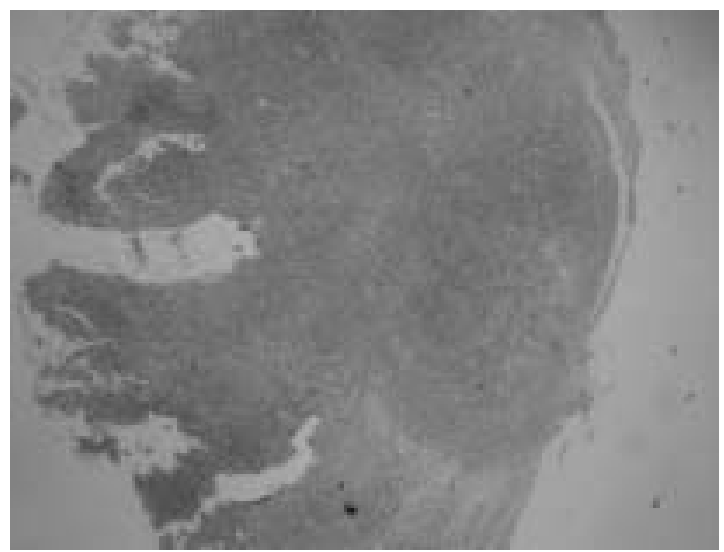

Figura 1. Aumento menor. Hematoxilina-Eosina. Muestra de biopsia cervical tomada con biótomo. Se observa estroma infiltrado homogéneamente por neoplasia.

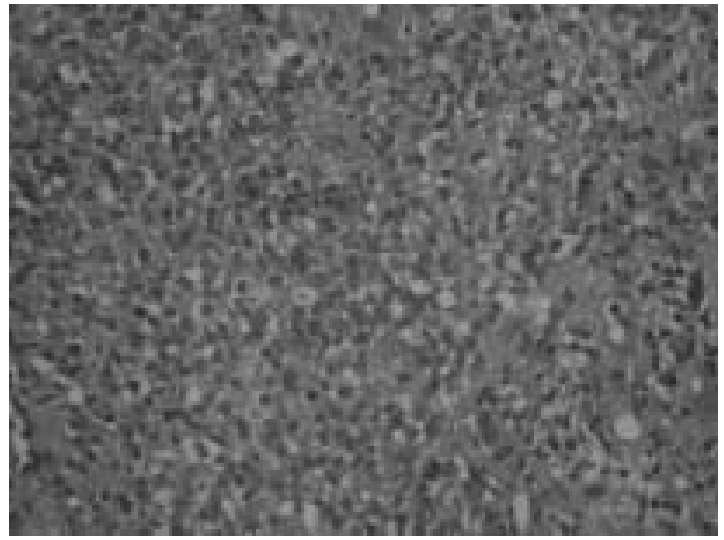

Figura 2. Aumento mayor (400x). Inmunohistoquímica para CD20, que muestra tinción de membrana en todas las células neoplásicas.

reveló la presencia de una gran masa cervical de $8 \mathrm{~cm}$ de diámetro mayor. Se tomó biopsia de la lesión informándose como linfoma no-Hodgkin (Figura 1). El estudio inmunohistoquímico mostró positividad para CD20 y negatividad para CD3 (Figura 2). El diagnóstico definitivo fue linfoma noHodgkin cervical primario difuso mixto de células grandes y pequeñas, estirpe $B$, de acuerdo a la clasificación de la WHO (Figura 3). La TAC de tórax, abdomen y pelvis mostró el tumor primario, y un conglomerado de adenomegalias pélvicas derechas. La biopsia de médula ósea fue negativa para infiltración medular. Se etapificó como II-AE según la clasificación de Ann-Arbor. Comenzó quimioterapia neoadyuvante con esquema m-CHOP cada 28 días por 4 ciclos. TAC abdominal y

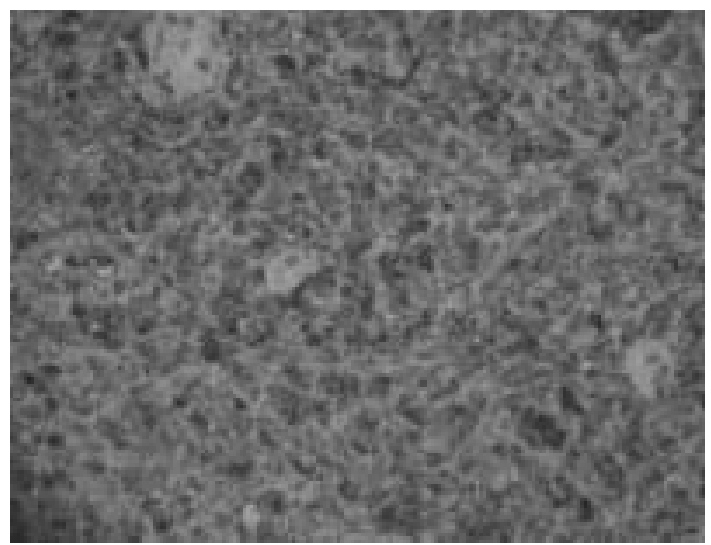

Figura 3. Aumento mayor (400x). Hematoxilina-Eosina. Tumor de elementos linfoides, mayoritariamente de gran tamaño. 
pelviano mostró remisión completa del tumor, lo que fue corroborado mediante examen clínico. Según lo planificado, recibió 3600 cGy a pelvis mediante radioterapia externa con Cobalto 60 (técnica de "four-field box"). Se mantiene sin evidencia de enfermedad a 23 meses del diagnóstico inicial.

\section{DISCUSIÓN}

Solo un tercio de los linfomas es extranodal (3). Los linfomas primarios en genitales son una rareza. Uno de cada 175 linfomas extranodales tiene su origen en la vagina, cuerpo o cuello uterino (1). El cuello uterino es el origen más frecuente entre éstos últimos, a la vez que el ovario es el sitio de metástasis de linfomas más frecuentes en el aparato genital femenino (2-4). Se ha calculado que entre $0,12-0,6 \%$ de todos los linfomas noHodgkin primarios extranodales se originan en el cuello uterino $(2,5,10)$. La edad de presentación está generalmente ubicada en la cuarta década de la vida $(6,10)$. La histología más frecuente es el linfoma no-Hodgkin, de estirpe $B$, difuso de células grandes $(3,7,8)$. El motivo de consulta más frecuente es el flujo genital anormal, casi invariablemente sanguinolento $(1,3,5)$. Macroscópicamente se presenta como una lesión de rápido crecimiento, acompañada por una sensación de presión u ocupamiento pélvico (6). Aunque por lo general estas neoplasias son de mayor tamaño al diagnóstico que otras, el diagnóstico diferencial clínico debe incluir al carcinoma escamoso (especialmente en sus presentaciones tipo "barrilete"), mioma cervical, sarcoma cervical y carcinoma de células pequeñas $(1,5)$. Dado que rara vez presentan ulceración, la citología cervical suele ser de poca utilidad (4). Todo esto hace que la biopsia sea el único procedimiento indispensable para el diagnóstico. Además de un examen físico exhaustivo en busca de adenomegalias periféricas sospechosas, el estudio de extensión debiera incluir siempre una biopsia de médula ósea y una TAC de tórax, abdomen y pelvis.

El tratamiento del linfoma primario del cuello uterino ha sido discutido por largo tiempo. La radioterapia sola o en combinación con quimioterapia o cirugía radical fue el primer enfoque terapéutico publicado $(3,4,6,9)$. Más recientemente se ha prestado atención a la quimioterapia combinada neoadyuvante sola o seguida de cirugía $(1,3$, $4,5,8,9)$. Además de preservar la fertilidad en muchos casos, la quimioterapia combinada tiene la ventaja de reducir la masa tumoral, lo que hace más fácil una eventual cirugía. Además podría prevenir la ocurrencia de micrometástasis al tiempo que preserva la función ovárica, factor crítico a considerar en la paciente joven $(4,5)$. A pesar de la edad de ambas pacientes, y del hecho de que se observó respuesta clínica e imagenológica completa tras la quimioterapia combinada, ambas completaron su tratamiento con radiación. Esto lo justificamos por 2 motivos principales:

a) Ambas pacientes solicitaron un tratamiento definitivo para su neoplasia, aún cuando su potencial reproductivo y/o función ovárica se viera comprometido, y;

b) Pensamos que no hay reporte de un suficiente número de pacientes tratadas con linfoma primario del cuello uterino como para poder considerar adecuada y segura la quimioterapia combinada sola en comparación con los esquemas más tradicionales de tratamiento (cirugía y/o radioterapia).

El pronóstico de la enfermedad se relaciona con la etapa. La importancia pronóstica de los distintos tipos histológicos y de las diversas modalidades de tratamiento no está aun definida. En una de las escasas publicaciones referentes al tema, la sobrevida de 18 casos fue de un $77 \%$ a 5 años (2). El pronóstico se considera bueno incluso en etapas avanzadas, entendiéndose por ello tumores T2 o T3.

Recientemente, se han reportado resultados alentadores en el tratamiento de linfomas noHodgkin con rituximab, que es un anticuerpo monoclonal dirigido hacia el antígeno CD20, que es expresado por la mayoría de estas neoplasias. A pesar de que se ha reportado su uso en linfomas no-Hodgkin asociados al Virus de la Inmunodeficiencia Humana $(\mathrm{VIH})$ y en el contexto de la terapia antiretroviral altamente activa (HAART), los resultados indican que probablemente esta sea la herramienta siguiente en el manejo de estas neoplasias (7).

\section{BIBLIOGRAFÍA}

1. Gabriele A, Gaudiano L. Primary malignant lymphoma of the cervix. A case report. J Reprod Med 2003; 48(11): 899-901.

2. Harris NL, Scully RE. Malignant lymphoma and granulocytic sarcoma of the uterus and vagina: A clinicopathologic analysis of 27 cases. Cancer 1984; 53: 2530-45.

3. Johnston C, Senekjian EK, Ratain MJ, Talerman A. Conservative management of primary cervical lymphoma using combination chemotherapy: a case report. Gynecol Oncol 1989 Dec; 35(3): 391-4. 
4. Sandvei R, Lote K, Svendsen E, Thunold S. Successful pregnancy following treatment of primary malignant lymphoma of the uterine cervix. Gynecol Oncol 1990; 38(1): 128-31.

5. Kuo HC, Chou CY, Chang CH, Liu MT, Tzeng CC, Huang KE. Primary malignant lymphoma of the uterine cervix shows favorable response to neoadjuvant chemotherapy. Gynecol Oncol 1994; 52(3): 40810.

6. Awwad JT, Khalil AM, Shamseddine AI, Mufarrij AA. Primary malignant lymphoma of the uterine cervix: is radiotherapy the best therapeutic choice for stage IE? Gynecol Oncol 1994; 52(1): 91-3.

7. Klepfish A, Schattner A, Shvidel L, Shtalrid M, Haran $M$, Bentwich Z, Berrebi A. Successful treatment of aggressive HIV-associated non-Hodgkin's lymphoma with combination chemotherapy, biotherapy with rituximab and HAART: presentation of a therapeutic option. Leuk Lymphoma 2003; 44(2): 349-51.

8. Pham DC, Guthrie TH, Ndubisi B. HIV-associated primary cervical non-Hodgkin's lymphoma and two other cases of primary pelvic non-Hodgkin's lymphoma. Gynecol Oncol 2003; 90(1): 204-6.

9. Szantho A, Balega JJ, Csapo Z, Sreter LL, Matolcsy A, Papp Z. Primary non-Hodgkin's lymphoma of the uterine cervix successfully treated by neoadjuvant chemotherapy: case report. Gynecol Oncol 2003; 89(1): 171-4.

10. Cheong IJ, Kim SH, Park CM. Primary Uterine Lymphoma: A Case Report. Korean J Radiol 2000; 1(4): 223-5. 